\title{
K-Ras and mitochondria: Dangerous liaisons
}

\author{
Jiri Neuzil $^{1,2}$, Jakub Rohlena ${ }^{2}$, Lan-Feng Dong ${ }^{1}$ \\ ${ }^{1}$ Apoptosis Research Group, School of Medical Science and the Griffith Health Institute, Griffith University Gold Coast Campus, \\ Southport, 4222 Qld, Australia; ' Institute of Biotechnology, Academy of Sciences of the Czech Republic, Prague, Czech Republic \\ Cell Research (2012) 22:285-287. doi:10.1038/cr.2011.160; published online 27 September 2011
}

It is well documented that the $K$ $R A S$ oncogene efficiently transforms non-malignant cells, and there is some evidence for the role of mitochondria in this process. Now Peng Huang and colleagues show that K-Ras induction results early on in mitochondria assuming the phenotype consistent with the so-called Warburg effect, i.e., increased glycolysis and attenuated oxidative phosphorylation. Thus the K-Ras protein capable of swift induction of phenotypic changes typical of cancer cells, yet these changes are reversible, and for cells to irreversibly reach their full malignant potential a much longer K-Ras expression is required, implicating mitochondria in the longer-term effects of the oncogene.

Malignant transformation has the dubious status of being the pivotal event during cancer development [1]. With cancer posing a continuous threat to the mankind and advancing even in the "non-traditional areas" [2], it is imperative that the process of malignant transformation is understood and eventually harnessed, however unlikely that may seem in the near future. It has been almost 30 years since the Ras family of oncogenes was discovered and described [3-5], yet the precise molecular

Correspondence: Jiri Neuzil

Tel: +61-2-55529109; Fax: +61-2-555-28444

E-mail: j.neuzil@griffith.edu.au mechanism(s) of the events triggered by the product of these oncogenes have not been clearly elucidated. So far, the best understood aspect of oncogenic K-Ras transformation is the upregulation of the MAP kinase signaling pathway to promote uncontrolled proliferation of cancer cells $[1,6]$.

Recently, another aspect of the Ras protein family in malignant transformation started to emerge. Several papers provided a strong link between Ras, mitochondria and the Bcl-2 family proteins. The mitochondrial connection is particularly exciting, since these DNAcontaining organelles are the powerhouse of the cells as well as the central purveyors of apoptotic cell death, and major sites of reactive oxygen species (ROS) generation [7, 8]. This is of importance given that mitochondria and mitochondrial formation of ROS are instrumental for malignant transformation mediated by increased expression of the oncogenic K-Ras [9]. In addition, as observed by Otto Warburg almost a century ago, malignant cells often alter their metabolic profile towards increased glycolysis and glucose consumption, a paradigm known as "Warburg effect" [10]. This has been disregarded for many years with oncogenes being at the forefront of the cancer-related research, but recently a clear connection started to emerge between oncogenic transformation and the metabolic shift described by Warburg. Conversely and reenforcing the inter-relationship between cell transformation and a variation in metabolism, glucose deprivation promotes K-Ras-dependent malignant transformation [11]. The involvement of mitochondria in the transformation and metabolic re-programming lies at hand, and this makes their connection with the $R A S$ oncogenes particularly interesting, as recently highlighted [8].

Huang and colleagues have made a great effort to further our understanding of the nitty-gritty of the events that lie at the heart of the mitochondria-oncogene connection, and this is the main thrust in their excellent publication in this issue of Cell Research [12]. The novelty of their approach lies in the ability to turn on the overexpression of the oncogenic $K-R A S$ variant $K-R A S^{G 12 V}$ in HEK293 cells as required, using a doxycyclin (DOX)-controlled inducible expression system, allowing the temporal observation of the events of RAS-promoted transformation (previous studies used stable overexpression of RAS). These model cells, referred to by a somewhat mystical term T-Rex/K-Ras cells, were then tested in a variety of conditions to better understand the relatively shortterm changes following switching on of the $K-R A S$ oncogene.

The major effect of the K-Ras oncoprotein was the rapid alteration of the mitochondrial function. Addition of DOX caused the expression of the KRAS $^{\mathrm{G} 12 \mathrm{~V}}$ protein in less than $12 \mathrm{~h}$ with its maximum level at $24 \mathrm{~h}$. Quite surprisingly, as soon as the K-Ras protein 
was discernible (by western blotting), rather prominent, mitochondria-related alterations were detected. These include the dissipation of the mitochondria inner transmembrane potential by $\sim 50 \%$ as well as $\sim 50 \%$ reduction in the oxygen consumption and $50 \%$ increase in the generation of ROS, with elevated spontaneous apoptosis ensuing 2-3 days later. Intriguingly, too, K-Ras induction caused a considerable change in the expression pattern of some of the key mitochondrial complexes: downregulation of complex I and upregulation of complex II, and this was accompanied by downregulation of superoxide dismutase-2 (SOD2) and catalase, two important antioxidant enzymes, often expressed at low levels in malignant cells (indicating the onset of a phenotypical change: cancer cells require sustained increase in ROS to promote their mitogenic pathways). Importantly, removal of DOX from the media reverted the cells to their pre-induction state, documenting a neat plasticity of the events. In line with Warburg's aerobic glycolysis hypothesis, K-Ras expression made the cells utilize more glycolysis for energy generation. Lastly, the finding that inoculation of nude mice with T-Rex/K-Ras cells pre-incubated with DOX gave much more efficient rise to tumors than when the mice were inoculated with cells devoid of preincubation with the inducer endows the study with a very nice translational spin. The cells had to be pre-incubated with DOX for extended periods of time (>1 month), indicating that additional changes, following the almost instant alteration in the mitochondrial function, were needed to make them truly malignant (see timeline in Figure 1).

This very nice investigative work sheds considerably more light on the molecular events arising from expression of oncogenes, epitomised here by $K-R A S^{G 12 V}$. It also highlights the multifaceted nature of the Warburg effect. Not only may the glycolysis be enhanced by direct regulatory action of oncogenes and transcription factors such as HIF $1 \alpha$ or Myc, or by damaged respiration compromised by mitochondrial DNA mutations, but the RAS oncogene expression may also result in immediate respiration defects and ensuing upregulation of glycolysis. However, the causal link between the $\mathrm{K}$-Ras protein and these respiration alterations is still unclear (it is anticipated that the authors are already conducting the relevant experiments). The respiration changes and related mitochondrial alterations ought to occur as a direct response to the increased level of the oncoprotein, since the cells do not have the time to re-arrange their expression of critical genes. This notion is corroborated by the finding that the K-Ras protein localized, in line with some previous reports, to mitochondria, supporting the important role for mitochondria in the transformation process [13]. Interestingly, mitochondrial localization has also been previously detected for other relevant proteins, such as the transcription factor STAT3 [13]. On the practical side, even though the precise molecular mechanisms that underlie the Rasinduced changes in the expression pattern of complex I and II are unknown, this observation may provide guidance in the search for an optimal molecular target for mitochondria-directed anticancer drugs $[14,15]$. In conclusion, we believe that this beautiful piece of work will stimulate others (the authors of this Research Highlight are most definitely encouraged in their research efforts) in the unrelenting quest to better understand the all important mitochondria-linked changes associated with malignant transformation in the hunt for the "magic bullet", i.e., an efficient and selective anti-cancer drug.

\section{References}

1 Hanahan D, Weinberg RA. Hallmarks of cancer: the next generation. Cell 2011; 144:646-674

2 Jemal A, Bray F, Center MM, et al. Global cancer statistics. CA Cancer $J$ Clin 2011; 61:69-90.

3 Balmain A, Pragnell IB. Mouse skin carcinomas induced in vivo by chemical carcinogens have a transforming Harvey-ras oncogene. Nature 1983; 303:72-74.

4 Hall A, Marshall CJ, Spurr NK, Weiss RA. Identification of transforming gene in two human sarcoma cell lines as a new member of the ras gene family located on chromosome 1. Nature 1983; 
303:396-400.

5 Santos E, Martin-Zanca D, Reddy EP, et al. Malignant activation of a K-ras oncogene in lung carcinoma but not in normal tissue of the same patient. Science 1984; 223:661-664.

6 Dhillon AS, Hagan S, Rath O, Kolch W. MAP kinase signalling pathways in cancer. Oncogene 2007; 26:32793290 .

7 Gogvadze V, Orrenius S, Zhivotovsky B. Mitochondria in cancer cells: what is so special about them? Trends Cell Biol 2008; 18:165-173.

8 Ralph SJ, Rodríguez-Enríquez S, Neuzil J, et al. The causes of cancer revisited: "mitochondrial malignancy" and ROS-induced oncogenic transformation - why mitochondria are targets for cancer therapy. Mol Aspects Med 2010; 31:145-170.

9 Weinberg F, Hamanaka R, Wheaton WW, et al. Mitochondrial metabolism and ROS generation are essential for Kras-mediated tumorigenicity. Proc Natl Acad Sci USA 2010; 107:87888793.

10 Vander Heiden MG, Cantley LC, Thompson CB. Understanding the Warburg effect: the metabolic requirements of cell proliferation. Science 2009; 324:1029-1033.

11 Yun J, Rago C, Cheong I, et al. Glucose deprivation contributes to the development of KRAS pathway mutations in tumor cells. Science 2009; 325:15551559.

$12 \mathrm{Hu} \mathrm{Y,} \mathrm{Lu} \mathrm{W,} \mathrm{Chen} \mathrm{G,} \mathrm{et} \mathrm{al.} \mathrm{K-ras}{ }^{\mathrm{G} 12 \mathrm{~V}}$ transformation leads to mitochondrial dysfunction and a metabolic switch from oxidative phosphorylation to glycolysis. Cell Res 2012; 22:399-412.

13 Gough DJ, Corlett A, Schlessinger K, et al. Mitochondrial STAT3 supports Ras-dependent oncogenic transformation. Science 2009; 324:1713-1716.

14 Rohlena J, Dong L, Ralph S, Neuzil J. Anticancer drugs targeting the mitochondrial electron transport chain. Antioxid Redox Signal 2011; 15:29512974.

15 Dong LF, Low $\mathrm{P}$, Dyason $\mathrm{J}$, et al. $\alpha$-Tocopheryl succinate induces apoptosis by targeting ubiquinone-binding sites in mitochondrial respiratory complex II. Oncogene 2008; 27:43244335 . 